Check for updates

Cite this: RSC Adv., 2018, 8, 4057

Received 21st November 2017 Accepted 15th January 2018

DOI: 10.1039/c7ra12634d

rsc.li/rsc-advances

\section{Characterization of magnetic relaxation when biofunctionalized magnetic nano-particles are associated with biomarkers in the liquid state in biomedical applications}

\author{
Shu-Hsien Liao, (iD *a Han-Sheng Huang, ${ }^{a}$ Jean-Hong Chen, ${ }^{b}$ Yu-Kai Su ${ }^{a}$ \\ and Yuan-Fu Tong ${ }^{a}$
}

This study determined the characteristics of the time-dependent effective relaxation time $\tau_{\text {eff }}$ and magnetization $M$ when biofunctionalized magnetic nanoparticles (BMNPs) associated with biomarkers in a liquid immunoassay. Carcinoembryonic antigen (CEA) was used as the biomarker. BMNP is an anti-CEA that was coated onto dextran-coated $\mathrm{Fe}_{3} \mathrm{O}_{4}$ and labeled as $\mathrm{Fe}_{3} \mathrm{O}_{4}$-anti-CEA. The phase lag $\theta$ of $M$ with respect to the applied field $H$ was measured using a sensitive homemade alternative-current susceptometer, and $\theta$ was measured using lock-in detection. The results were used to estimate $\tau_{\text {eff }}$ using the relationship $\tan \theta(t)=\omega \tau_{\text {eff }}(t)$, where $2 \pi f=\omega$ and $f$ is the excitation frequency. $\Delta \tau_{\text {eff }}$ increased with $\Phi_{\text {CEA }}$, where $\Delta \tau_{\text {eff }}$ was the increment of $\tau_{\text {eff }}$ after $\mathrm{Fe}_{3} \mathrm{O}_{4}$-anti-CEA associated with CEA, and $\Phi_{\text {CEA }}$ was the concentration of CEA. Additionally, $M$ enhanced when $\Phi_{\text {CEA }}$ increased. We attributed these enhancements to magnetic dipole-dipole interactions among MNPs that contributed extra $M$ and in turn

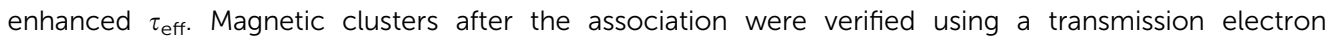
microscope. This study established the relationship between $\Delta \tau_{\text {eff }} / \tau_{\text {eff,O }}$ and $\Phi_{\text {CEA, }}$ where $\tau_{\text {eff,O }}=\tau_{\text {eff }}$ $\left(\Phi_{\text {CEA }}=0\right)$, allowing the assay of an unknown amount of CEA molecules.

\section{Introduction}

Immunoassays use a variety of labels for assaying antibodies or antigens. These labels are typically chemically conjugated or linked to desired analyses. The most common methods are enzyme-linked immunosorbent assay, ${ }^{1}$ radioimmunoassay, ${ }^{2,3}$ and real-time quantitative polymerase chain reaction. ${ }^{4}$ Recently, magnetic immunoassays (MIAs) ${ }^{5,6}$ have gained considerable attention because there is negligible magnetic background in a biomolecular sample during magnetic detection; thus, MIAs can reach a high detection sensitivity. In MIAs, biofunctionalized magnetic nanoparticles (BMNPs) are used as labels. ${ }^{7}$ The assays involve the specific binding of a bioprobe to its analyte, and a magnetic label is conjugated to one element of the pair to form magnetic clusters. Because of molecular interactions between MNPs, their magnetic properties change after the association. These changes in magnetic characteristics provide a sensing mechanism. The signal for magnetic clusters is then detected using magnetic sensors, which measure the change of magnetic signals that is induced by magnetic clusters. Changes in magnetic signals are proportional to the

${ }^{a}$ Institute of Electro-Optical Science and Technology, National Taiwan Normal University, Taipei 116, Taiwan.E-mail: shliao@ntnu.edu.tw

${ }^{b}$ Department of Materials Engineering, Kun Shan University, Tainan 710, Taiwan concentrations of analytes (e.g., viruses, bacteria, tumor markers, and cardiac markers) in samples. Different sensing mechanisms have been used for MIAs, such as Brownian relaxation time, ${ }^{8}$ Néel relaxation time, ${ }^{9-12}$ remanent magnetization, ${ }^{13,14}$ saturation magnetization, ${ }^{15,16}$ spin-spin relaxation time,${ }^{17}$ and alternative-current (AC) susceptibility. ${ }^{18-26}$

Effective relaxation time $\tau_{\text {eff }}$ is one of the magnetic properties that are affected by the magnetic clustering of BMNPs when they associate with biomarkers. The value of $\tau_{\text {eff }}$ reflects the effect of clustered MNPs on Brownian and Néel relaxations. ${ }^{27}$ To study $\tau_{\text {eff }}$, Liao et al. used a homemade AC susceptometer. ${ }^{28}$

In the current study, a sensitive homemade AC susceptometer was used to characterize $\tau_{\text {eff }}$ when BMNPs that are composed of $\mathrm{Fe}_{3} \mathrm{O}_{4}$-anti-carcinoembryonic antigen (CEA) associated with CEA in biomedical applications. This study found that $\Delta \tau_{\text {eff }}$ increased with the concentration of CEA, $\Phi_{\text {eff }}$, where $\Delta \tau_{\text {eff }}$ was the increment of $\tau_{\text {eff }}$. This increase was attributed to dipole-dipole interactions between MNPs, which contributed extra magnetization and in turn increased the value of $\tau_{\text {eff. }}$ A relationship between $\Delta \tau_{\text {eff }} / \tau_{\text {eff,o }}$ and $\Phi_{\text {CEA }}$ was established, where $\tau_{\text {eff }, 0}=\tau_{\text {eff }}\left(\Phi_{\text {CEA }}=0\right)$. This relationship is based on a $\left(\Delta \tau_{\text {eff }} / \tau_{\text {eff, } 0}\right)$-versus $-\Phi_{\text {CEA }}$ curve and can be used to determine an unknown amount. This paper also discusses the merits of using the $\Delta \tau_{\text {eff }} / \tau_{\text {eff, } 0}$ ratio for biosensing. 


\section{Experiments}

BMNPs are anti-CEAs that were coated onto dextran-coated MNPs and labeled as $\mathrm{Fe}_{3} \mathrm{O}_{4}$-anti-CEA (MF-CEA-0060, MagQu Co., Taiwan). The biotarget was CEA (AbD). A feasibility study for clinical use reported the development of antibodyfunctionalized magnetic nanoparticles for the immunoassay of CEA. ${ }^{29}$ The mean diameter of BMNPs was $53.8 \pm 17.8 \mathrm{~nm},{ }^{23}$ as detected through dynamic light scattering (Nanotrac-150, Microtrac) and shown in Fig. 1a. The magnetization reagent was characterized using a vibrating sample magnetometer (Hystermag MagQu Co. Taiwan). The magnetic clustering after association was measured using a field-emission transmission electron microscope (Philips Tecnai F20 G2). Before being examined, the reagent consisted of magnetic clusters that were dispersed in an extremely diluted solvent $(1: 50)$. The samples were then dried under vacuum at room temperature for $48 \mathrm{~h}$.

Fig. 1b shows pictures of CEA, anti-CEA, functionalized $\mathrm{Fe}_{3} \mathrm{O}_{4}$-anti-CEA, and a magnetic cluster that is composed of $\mathrm{Fe}_{3} \mathrm{O}_{4}$-anti-CEA-CEA. The magnetic clusters of $\mathrm{Fe}_{3} \mathrm{O}_{4}$-anti-CEACEA conjugated when CEAs were mixed with a reagent that consisted of functionalized $\mathrm{Fe}_{3} \mathrm{O}_{4}$-anti-CEA.

\section{AC susceptibility}

The AC susceptibility $\chi_{\mathrm{ac}}(\omega)$ of the samples is expressed as follows: $:^{28,30}$

$$
\chi_{\mathrm{ac}}=\chi^{\prime}+i \chi^{\prime \prime}
$$

with

$$
\begin{gathered}
\chi^{\prime}=\chi_{0}\left\{1 /\left[1+\left(\omega \tau_{\text {eff }}\right)^{2}\right]\right\}, \\
\chi^{\prime \prime}=\chi_{0}\left(\omega \tau_{\text {eff }}\right) /\left\{\left[1+\left(\omega \tau_{\text {eff }}\right)^{2}\right]\right\}
\end{gathered}
$$

where $i=(-1)^{1 / 2}, \theta=\tan ^{-1} \omega \tau_{\mathrm{eff}}(t)=\chi^{\prime \prime} / \chi^{\prime}$ is the phase lag for the time-varying magnetization, $M(t)$, with respect to the applied AC magnetic field, $H(t)$, and $\tau_{\text {eff }}$ is the effective relaxation time. In this study, $\theta(t)$ was detected using a homemade AC susceptometer and the lock-in detection technique at $18 \mathrm{kHz}$. The time evolution of $\tau_{\text {eff }}(t)$ was measured by monitoring $\theta(t)$. The value of $\tau_{\text {eff }}(t)$ was estimated using the relationship $\theta=$ $\tan ^{-1} \omega \tau_{\text {eff }}(t)$. The value of $\Delta \tau_{\text {eff }}=\left[\tau_{\text {eff }}(t=0)-\tau_{\text {eff }}(t=\infty)\right]$ was given as a function of the concentration of biomarkers to characterize their abundance.

\section{Results and discussion}

To explore and confirm the combination of $\mathrm{Fe}_{3} \mathrm{O}_{4}$-anti-CEA and CEA, a TEM was used to verify the presence of magnetic clustering when CEA associated with $\mathrm{Fe}_{3} \mathrm{O}_{4}$-anti-CEA. Fig. 2a shows a TEM image of CEA molecules; single CEA molecules are marked with small dashed circles. A CEA molecule has a mean length of approximately $29 \mathrm{~nm},{ }^{31}$ which is close to the value given in Fig. 2b. Fig. 2b shows a TEM image of magnetic clusters when $\mathrm{Fe}_{3} \mathrm{O}_{4}$-anti-CEA associated with CEA molecules; some magnetic clusters are marked with a large dashed circle. Each magnetic cluster was composed of several small magnetic clusters. Also marked is the independent $\mathrm{Fe}_{3} \mathrm{O}_{4}$-anti-CEA and MNPs inside the magnetic clusters; the formation of magnetic clusters was verified using a TEM. The size of associations with CEA molecules was changeable; if the concentration of CEA molecules was higher, then a magnetic cluster was formed. Thus, the size of $\mathrm{Fe}_{3} \mathrm{O}_{4}$-anti-CEA associated with CEA molecules increased.

Fig. 3 illustrates curves for $M$ as a function of $H$ after the reagents that were composed of $\mathrm{Fe}_{3} \mathrm{O}_{4}$-anti-CEA associated with different concentrations of CEA in a liquid state. This shows that when $H=0.16 \mathrm{~T}$ and $M=0.07 \mathrm{emu} \mathrm{g}^{-1}$ for the reagent, then $M$ increased to $0.011,0.154$, and $0.218 \mathrm{emu}^{-1}$ after the (a)

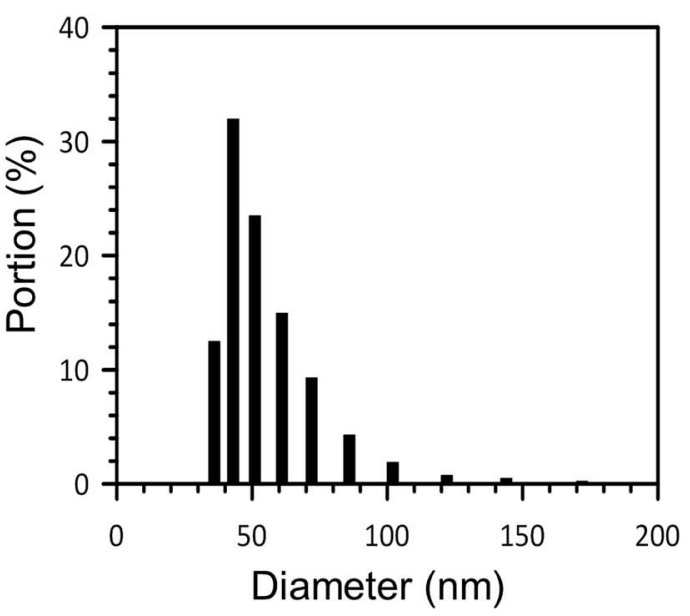

(b)

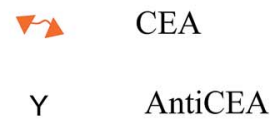

(3)

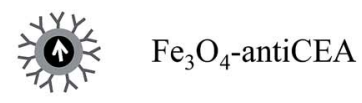

(4)

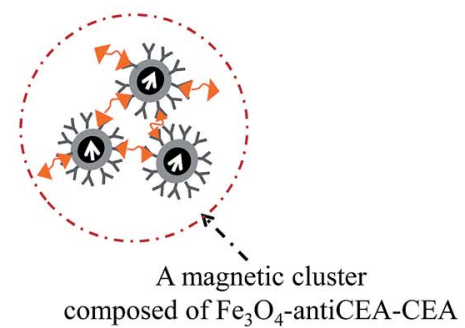

Fig. 1 (a) The size distribution diagram of BMNPs. (b) A representation of CEA, $\mathrm{Fe}_{3} \mathrm{O}_{4}$-anti-CEA, and a magnetic cluster composed of $\mathrm{Fe}_{3} \mathrm{O}_{4}$-anti-CEA-CEA. 
(a)

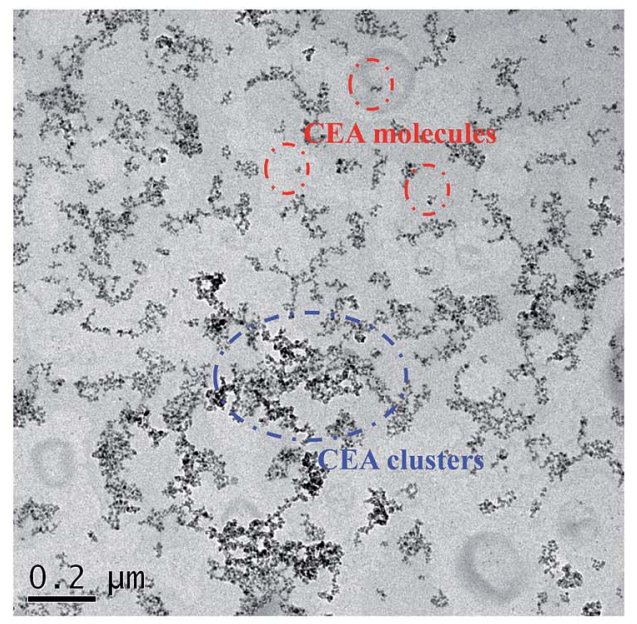

(b)

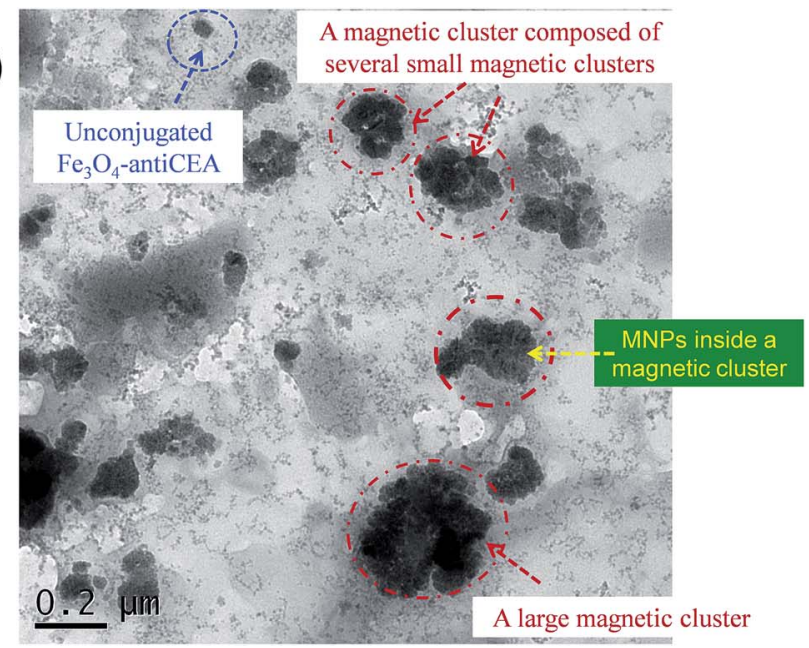

Fig. 2 (a) A TEM picture of CEA molecules and (b) magnetic clusters after $\mathrm{Fe}_{3} \mathrm{O}_{4}$-anti-CEA associated with CEA molecules.

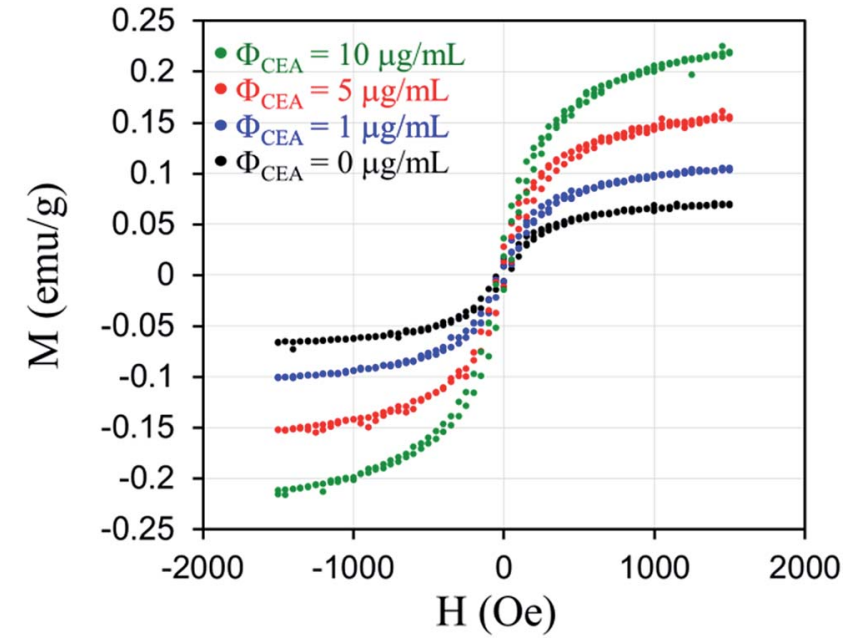

Fig. 3 Curves for $M$ as a function of $H$ after the reagents that are composed of $\mathrm{Fe}_{3} \mathrm{O}_{4}$-anti-CEA associated with different concentrations of CEA in a liquid state.

reagent with $\Phi_{\mathrm{CEA}}=1,5$, and $10 \mu \mathrm{g} \mathrm{mL}^{-1}$ associated with CEA, respectively. This increase is attributable to dipole-dipole interactions between MNPs in the magnetic clusters. Thus, the magnetization increased with the CEA concentration.

For a homogeneous MNP with a total number of particles, $N$, a mean magnetic moment, $\langle m\rangle$, and an effective mean diameter, $\langle d\rangle_{\text {eff }}$, the magnetization $M(H)$ at $H$ is expressed as follows:

$$
M(H)=N\langle m\rangle L[(\alpha H)]=\alpha L[(\beta H)]
$$

and

$$
L(x)=\operatorname{coth}(x)-1 / x
$$

where $M_{\text {sat }}=N\langle m\rangle$ and $\beta=\mu_{\mathrm{o}}\langle m\rangle / k_{\mathrm{B}} T, k_{\mathrm{B}}$ is Boltzmann's constant and $T$ is the absolute temperature (a distribution of magnetic nanoparticles, the magnetic properties, such as the magnetization, are detailed in the ref. 32).
Fig. 4a exhibits the change in $\theta$ over time when $\mathrm{Fe}_{3} \mathrm{O}_{4}$-antiCEA associated with different CEA concentrations. $\Phi_{\text {CEA }}$ was detected using an AC susceptometer and a lock-in technique. $\theta$ increased over time and $\theta=6^{\circ}$ for the reagent. The value of $\theta$ increased to $\theta=9.4^{\circ}$ when $t=7200 \mathrm{~s}$ for CEA with a $\Phi_{\mathrm{CEA}}=10$ $\mu \mathrm{g} \mathrm{mL}{ }^{-1}$ conjugated with $\mathrm{Fe}_{3} \mathrm{O}_{4}$-anti-CEA. Fig. $4 \mathrm{~b}$ shows the relationship between $\tau_{\text {eff }}$ and time for the reaction process when the reagent conjugated with different concentrations of $\Phi_{\text {CEA }}$. The value of $\tau_{\text {eff }}$ for the reagent was $0.93 \mu \mathrm{s}$ when $t=0$ and remained constant until $t=7200 \mathrm{~s}$, which demonstrated that the AC susceptometer had stable performance. In the reaction with $10 \mu \mathrm{g} \mathrm{mL}^{-1} \mathrm{CEA}$, the value of $\tau_{\text {eff }}$ was approximately $0.93 \mu \mathrm{s}$ when $t=0$ and the value of $\tau_{\text {eff }}$ increased to $1.43 \mu$ s when $t=$ $7200 \mathrm{~s}$. The $\tau_{\text {eff }}$-versus- $t$ curve showed saturation at approximately $t=7200 \mathrm{~s}$.

Yang et al. studied the effect of temperature on immunoreaction kinetics for a BMNP assay of biomarkers for colorectal cancer. ${ }^{33}$ The activation energy that was required for the reaction in immunoassay was determined to be $10^{-19} \mathrm{~J}$ per molecule. At higher temperatures, a significant increase was observed in the number of molecules with energy that was higher than the activation energy, which is the principal reason for the increase in the reaction rate. The current study indicated that it takes approximately $2 \mathrm{~h}$ to complete the association at 298 K. An increase in the reaction temperature decreased the reaction time.

Fig. 5 illustrates the value of $\tau_{\text {eff }}$ as a function of $\Phi_{\text {CEA }}$ when the reagent associated with various concentrations of CEA; the red line is a guide. It is seen that $\tau_{\text {eff }}=0.93 \mu$ s for a reagent that consisted of $\mathrm{Fe}_{3} \mathrm{O}_{4}$-anti-CEA. The value of $\tau_{\text {eff }}$ increased to $1.43 \mu \mathrm{s}$ when $\Phi_{\mathrm{CEA}}=10 \mu \mathrm{g} \mathrm{\textrm {mL } ^ { - 1 }}$ associated with the reagent. The increase in the value of $\tau_{\text {eff }}$ after association with $\mathrm{Fe}_{3} \mathrm{O}_{4}$-anti-CEA is due to magnetic interactions between MNPs in the associated magnetic clusters. The value of $M$ increased; therefore, the value of $\tau_{\text {eff }}$ increased.

Two simultaneous relaxations occur during the reaction process: Néel and Brownian relaxation. In Brownian relaxation, 
(a)

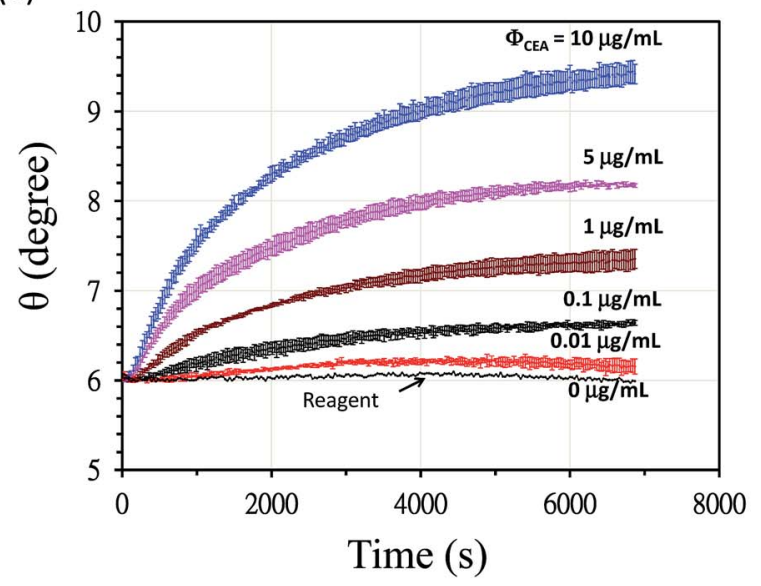

(b)

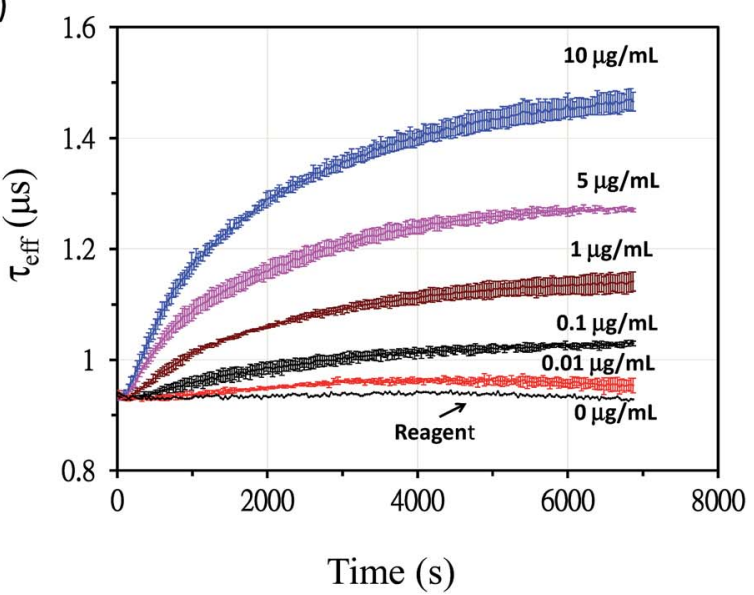

Fig. 4 (a) The relationship between $\theta$ for CEA and time in the reaction process and (b) the relationship between $\tau_{\text {eff }}$ and time when the reagent associated with different concentrations of CEA.

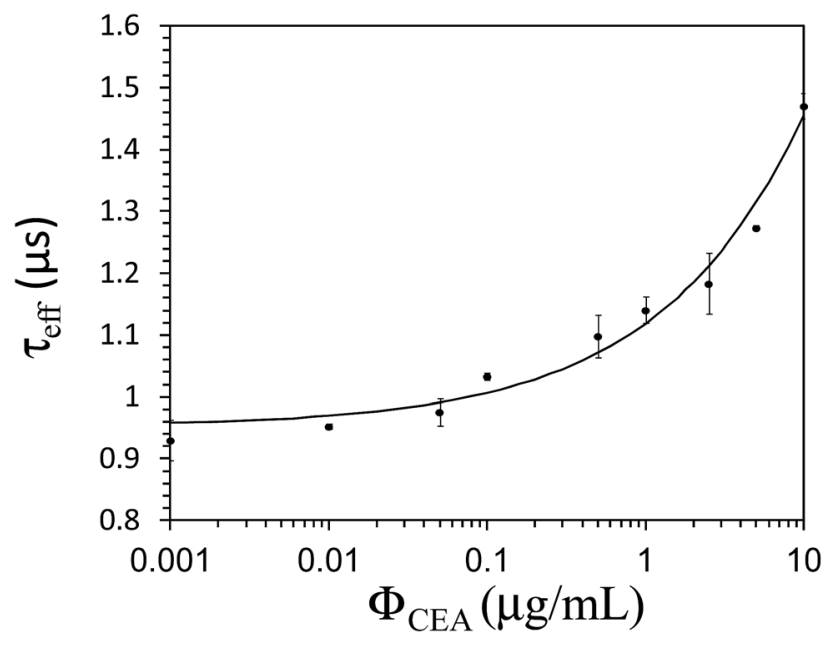

Fig. $5 \tau_{\text {eff }}$ as a function of $\Phi_{\text {CEA }}$.

the magnetic moment is locked to the crystal axis and the particle rotates. The corresponding relaxation time is $\tau_{\mathrm{B}}$ when the field is turned off. In Néel relaxation, the magnetic moment rotates within the crystal, and the corresponding relaxation time is $\tau_{\mathrm{N}}$. The Brownian relaxation time $\tau_{\mathrm{B}}$ is expressed as $\tau_{\mathrm{B}}=$ $3 \eta V_{\mathrm{H}} / k_{\mathrm{B}} T,{ }^{29}$ where $\eta$ is the viscosity coefficient, $k_{\mathrm{B}}=1.38 \times$ $10^{-23} \mathrm{~J} \mathrm{~K}^{-1}$ is the Boltzmann constant, $T$ is the absolute temperature (K), and $V_{\mathrm{H}}$ is the hydrodynamic volume of BMNPs. The Néel relaxation time, $\tau_{\mathrm{N}}$, is given by the expression, $\tau_{\mathrm{N}}=$ $\left[(\pi)^{1 / 2} / 2\right] \tau_{0} \exp [\rho] /(\rho)^{1 / 2}$, where $\rho=K V_{\mathrm{M}} / k_{\mathrm{B}} T, K$ is the anisotropy constant, $\tau_{0}$ is the relaxation time, and $V_{\mathrm{M}}=(4 \pi / 3) R^{3}$ is the magnetic volume of MNPs with radius $R$. The approximate mean core value was $\langle R\rangle=37 \mathrm{~nm}$ in this study. If $\langle R\rangle=37 \mathrm{~nm}$, then $\tau_{\text {Brownian }}$ is in the order of microseconds and $\tau_{\text {Néel }}$ is in the order of milliseconds or longer. ${ }^{27}$ The mean hydrodynamic diameter of BMNPs in this work was approximately $47 \mathrm{~nm}$, which equates to a core size of approximately $38 \mathrm{~nm}$. Because $1 / \tau_{\text {eff }}=1 / \tau_{\text {Brownian,eff }}+1 / \tau_{\text {Néel,eff }}$ and $1 / \tau_{\text {Brownian,eff }} \gg 1 / \tau_{\text {Néel,eff }}$, the values of $1 / \tau_{\text {eff,eff }} \sim 1 / \tau_{\text {Brownian,eff }}$ can be approximated.
Brownian relaxation dominates the time dependency of $\tau_{\text {eff }}$ in the association process that is shown in Fig. $4 \mathrm{~b}$.

Fig. 6 illustrates $\Delta \tau_{\text {eff }} / \tau_{\text {eff }, 0}$ as a function of $\Phi_{\text {CEA }}$ when $\mathrm{Fe}_{3} \mathrm{O}_{4}$-anti-CEAs conjugated with CEAs, where $\Delta \tau_{\text {eff }}=\tau_{\text {eff }}\left(\Phi_{\text {CEA }}\right)$ $-\tau_{\text {eff }}\left(\Phi_{\text {CEA }}=0\right)$ and $\tau_{\text {eff }, 0}=\tau_{\text {eff }}\left(\Phi_{\text {CEA }}=0\right)$ at $t=7200 \mathrm{~s}$. In an assay of $10 \mu \mathrm{g} \mathrm{mL} \mathrm{m}^{-1}$ CEA molecules, $\Delta \tau_{\text {eff }} / \tau_{\text {eff }, 0}=0.05$ as $\Phi_{\text {CEA }}$.

Fig. $6 \Delta \tau_{\text {eff }} / \tau_{\text {eff }, 0}$ as a function of $\Phi_{\text {CEA }}=0.01 \mu \mathrm{g} \mathrm{mL}^{-1}$ and $\Delta \tau_{\text {eff }} / \tau_{\text {eff,o }}$ increases to 0.54 when $\Phi_{\text {CEA }}=0.01 \mu \mathrm{g} \mathrm{mL}{ }^{-1}$; $\Delta \tau_{\text {eff }} / \tau_{\text {eff }, 0}$ increases when $\Phi_{\text {CEA }}$ increases.

The value of $\Delta \tau_{\text {eff }} / \tau_{\text {eff }, 0}$ as a function of $\Phi_{\mathrm{CEA}}$ is described by a logistic function: ${ }^{34}$

$$
\Delta \tau_{\mathrm{eff}} / \tau_{\mathrm{eff}, 0}=(A-B) /\left\{1+\left[\left(\Phi_{\mathrm{CEA}}\right) /\left(\tau_{0}\right)\right]^{\gamma}\right\}+B
$$

where $\Delta \tau_{\text {eff }}=\tau_{\text {eff }}\left(\Phi_{\mathrm{CEA}}=0\right)$ and $\tau_{\text {eff }, 0}=\tau_{\text {eff }}\left(\Phi_{\mathrm{CEA}}=0\right)$. The solid line is a fitting curve to eqn (6), with fitting parameters $A=0.03$, $B=13.9, \Phi_{0}=7243 \mu \mathrm{g} \mathrm{mL}{ }^{-1}$, and $\gamma=0.49$. The relationship between $\Delta \tau_{\text {eff }} / \tau_{\text {eff }, 0}$ and $\Phi_{\mathrm{CEA}}$ was established. This relationship, $\left(\Delta \tau_{\text {eff }} / \tau_{\text {eff, }, 0}\right)$-versus- $\Phi_{\text {CEA }}$, allows the assay of an unknown amount

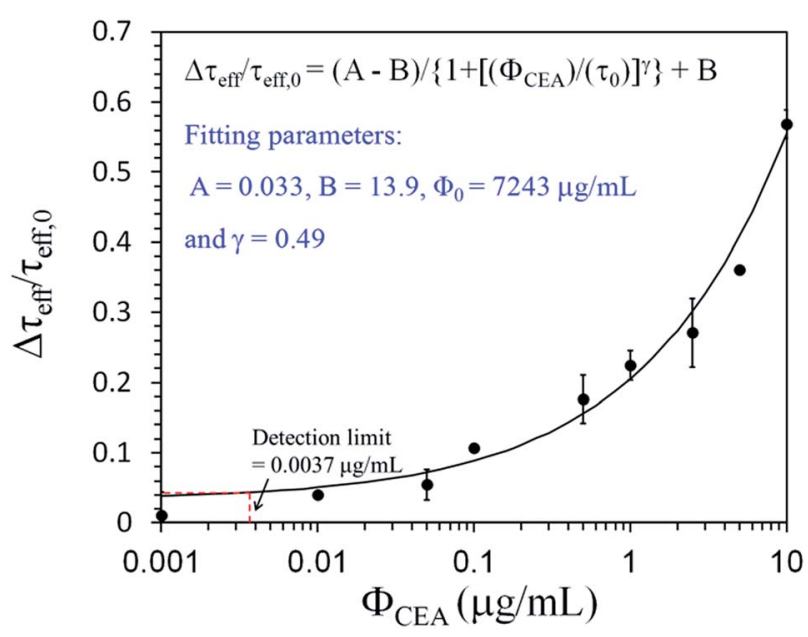

Fig. $6 \Delta \tau_{\text {eff }} / \tau_{\text {eff, } 0}$ as a function of $\Phi_{\text {CEA }}$. 
of CEA molecules for biosensing. Colorectal tumors, which are caused by uncontrolled cell growth in the colon or rectum, ${ }^{35}$ are the most commonly diagnosed cancer, especially in developed countries. ${ }^{36}$ Quantitative measures of CEA molecules are used as a tumor marker to monitor colorectal carcinoma treatment, to identify recurrences after surgical resection, and to stage or localize the spread of cancer. The detection sensitivity in this study can be determined from the variation of $\Delta \tau_{\text {eff }} / \tau_{\text {eff, } 0}$. The standard deviation of $\Delta \tau_{\text {eff }} / \tau_{\text {eff }, 0}$ was 0.033 as $\Phi_{\text {CEA }}=0.001 \mu \mathrm{g}$ $\mathrm{mL}^{-1}$. The estimated detection limit of $0.0037 \mu \mathrm{g} \mathrm{mL}{ }^{-1}$ was obtained by the highest value of $\Delta \tau_{\text {eff }} / \tau_{\text {eff, } 0}$ and transferred using a logistic function. The detection limit in our system reached the detection criteria for colorectal carcinoma diagnosis.

\section{Conclusions}

This study determined the relationship between time and $\tau_{\text {eff }}$ when a reagent that consisted of $\mathrm{Fe}_{3} \mathrm{O}_{4}$-anti-CEA associated with CEA molecules. Because of magnetic interactions between MNPs in magnetic clusters, which contributed extra magnetization, Néel relaxation and Brownian relaxation increased, which resulted in an increase in the value of $\tau_{\text {eff. Magnetic }}$ clustering was verified using a TEM. A relationship between $\Delta \tau_{\text {eff }} / \tau_{\text {eff }, 0}$ and $\Phi_{\text {CEA }}$ was established, which obeyed a logistic function, allowing the assay of an unknown amount of CEA molecules. The $\chi_{\text {ac }}$ detection platform for sensing biomarkers is highly sensitive, easy to use, and can be used to assay a wide variety of analytes, such as proteins, DNA, viruses, and tumor markers.

\section{Conflicts of interest}

There are no conflicts to declare.

\section{Acknowledgements}

This work is supported by the Ministry of Science and Technology of Taiwan under grant number: 104-2112-M-003-005, 105-2112-M-003-012, 106-2112-M-003-002 and by "Aim for the Top University Plan” of the National Taiwan Normal University and the Ministry of Education, Taiwan, R. O. C under grant number 104J1A27 and 105J1A27.

\section{References}

1 E. Engvall and P. Perlmann, Enzyme-linked immunosorbent assay (ELISA) quantitative assay of immunoglobulin G, Immunochemistry, 1971, 8(9), 871-874, DOI: 10.1016/00192791(71)90454-X, PMID 5135623.

2 S. Acton, A. Rigotti, K. T. Landschulz, S. Xu, H. H. Hobbs and M. Krieger, Identification of scavenger receptor SR-BI as a high density lipoprotein receptor, Science, 1990, 271, 518520.

3 M. Cattet and J. R. Geraci, Distribution and elimination of ingested brevetoxin (PbTx-3) in rats, Texicon, 1993, 31, 1483-1486.
4 J. M. S. Bartlett and D. Stirling, A Short History of the Polymerase Chain Reaction, PCR Protocols, Methods in Molecular Biology, 2nd edn, 2003, vol. 226, pp. 3-6, DOI: 10.1385/1-59259-384-4:3, ISBN 1-59259-384-4.

5 C.-Y. Hong, C. C. Wu, Y. C. Chiu, S. Y. Yang, H. E. Horng and H. C. Yang, Magnetic Susceptibility Reduction Method for Magnetically Labeled Immunoassay, Appl. Phys. Lett., 2006, 88, 212512.

6 K. Enpuku, K. Soejima, T. Nishimoto, T. Matsuda, H. Tokumitsu, T. Tanaka, K. Yoshinaga, H. Kuma and N. Hamasaki, Biological immunoassays without bound/free separation utilizing magnetic marker and HTS SQUID, IEEE Trans. Appl. Supercond., 2007, 17, 816.

7 C. R. Tamanaha, S. P. Mulvaney, J. C. Rife and L. J. Whitman, Magnetic labeling, detection, and system integration, Biosens. Bioelectron., 2008, 24, 1-13, PMID 18374556.

8 K. Enpuku, H. Tokumitsu, Y. Sugimoto, H. Kuma, N. Hamasaki, A. Tsukamoto, T. Mizoguchi, A. Kandori, K. Yoshinaga, H. Kanzaki and N. Usuki, Fast Detection of Biological Targets With Magnetic Marker and SQUID, IEEE Trans. Appl. Supercond., 2009, 19, 844.

9 R. Kötitz, W. Weitschies, L. Trahms, W. Brewer and W. Semmler, J. Magn. Magn. Mater., 1999, 194, 62.

10 S. K. Lee, W. R. Myers, H. L. Grossman, H.-M. Cho, Y. R. Chemla and J. Clarke, Appl. Phys. Lett., 2002, 81, 3094.

11 W. Weitschies, T. Bunte and L. Trahms, Pharm. Pharmacol. Lett., 1997, 7, 1.

12 R. Kotitz, H. Matz, L. Trahms, H. Koch, W. Weitschies, T. Rheinlander, W. Semmler and T. Bunte, IEEE Trans. Appl. Supercond., 1997, 7, 3678-3681.

13 K. Enpuku, T. Minotani, T. Gima, Y. Kuroki, Y. Itoh, M. Yamashita, Y. Katakura and S. Kuhara, Jpn. J. Appl. Phys., 1999, 38, L1102.

14 K. Enpuku, K. Inoue, K. SoeJima, K. Yoshinaga, H. Kuma and N. Hamasaki, IEEE Trans. Appl. Supercond., 2005, 15, 660.

15 H. E. Horng, S. Y. Yang, C.-Y. Hong, C. M. Liu, P. S. Tsai, H. C. Yang and C. C. Wu, Appl. Phys. Lett., 2006, 88, 252506.

16 J.-J. Chieh, K.-W. Huang and J.-C. Shi, Sub-tesla-field magnetization of vibrated magnetic nanoreagents for screening tumor markers, Appl. Phys. Lett., 2015, 106, 073703.

17 H. Lee, E. Sun, D. Ham and R. Weissleder, Chip-NMR biosensor for detection and molecular analysis of cells, Nat. Med., 2008, 14, 869.

18 K.-W. Huang, J.-J. Chieh, H.-E. Horng, C.-Y. Hong and H.-C. Yang, Characteristics of magnetic labeling on liver tumors with anti-alpha-fetoprotein-mediated $\mathrm{Fe}_{3} \mathrm{O}_{4}$ magnetic nanoparticles, Int. J. Nanomed., 2012, 7, 2987.

19 C. C. Yang, S. Y. Yang, J. J. Chieh, H. E. Horng, C. Y. Hong and H. C. Yang, Universal behavior of bio-moleculeconcentration dependent reduction in ac magnetic susceptibility of bio-reagents, IEEE Magn. Lett., 2012, 3, 1500104.

20 S. Tanaka, H. Ota, Y. Kondo, Y. Tamaki, S. Kobayashi and S. Noguchi, IEEE Trans. Appl. Supercond., 2003, 13, 377-380.

21 K. Enpuku, T. Minotani, M. Hotta and A. Nakahodo, IEEE Trans. Appl. Supercond., 2001, 11, 661-664. 
22 K. Enpuku, Y. Tamai, T. Mitake, T. Yoshida and M. Matsuo, AC susceptibility measurement of magnetic markers in suspension for liquid phase immunoassay, J. Appl. Phys., 2010, 108, 034701.

23 K.-W. Huang, J.-J. Chieh, I.-T. Lin, H.-E. Horng, H.-C. Yang and C.-Y. Hong, Anti-CEA biofuntionalized superparamagnetic iron oxide nanoparticles for examining colorectal tumors in vivo, Nanoscale Res. Lett., 2013, 8, 413.

24 M.-J. Chiu, S.-Y. Yang, H.-E. Horng, C. Chuan Yang, Ta-F. Yang Chen, J.-J. Chieh, H.-H. Chen, T.-C. Chen, C.-S. Ho, S.-F. Chang, H. Chun Liu, C. Yih Hong and H.-C. Yang, Combined plasma biomarkers for diagnosing mild cognition impairment and Alzheimer's disease, ACS Chem. Neurosci., 2013, 4, 1530.

25 M. J. Chiu, S. Y. Yang, T. F. Chen, J. J. Chieh, T. Z. Huang, P. K. Yip, H. C. Yang, T. W. Cheng, Y. F. Chen, M. S. Hua and H. E. Horng, New assay for old markers-Plama beta amyloid of mild cognitive impairment and Alzheimer's disease, Curr. Alzheimer Res., 2012, 9, 1142.

26 S. Y. Yang, J. J. Chieh, C. C. Yang, S. H. Liao, H. H. Chen, H. E. Horng, H. C. Yang, C. Y. Hong, M. J. Chiu, T. F. Chen, K. W. Huang and C. C. Wu, Clinic Applications in Assaying Ultra-Low-Concentration Bio-Markers Using HTS SQUID-Based AC Magnetosusceptometer, IEEE Trans. Appl. Supercond., 2013, 23, 1600604.

27 R. E. Rosensweig and J. Magn, Magn. Mater., 2002, 252, 370374.

28 S. H. Liao, H. C. Yang, H. E. Horng, J. J. Chieh, K. L. Chen, H. H. Chen, J. Y. Chen, C. I. Liu, C. W. Liu and L. M. Wang, Time-dependent phase lag of biofunctionalized magnetic nanoparticles conjugated with biotargets studied with alternating current magnetic susceptometor for liquid phase immunoassays, Appl. Phys. Lett., 2013, 103, 243703.

29 C.-C. Yang, S.-Y. Yang, C.-S. Ho, J.-F. Chang, B.-H. Liu and K.-W. Huang, Development of antibody functionalized magnetic nanoparticles for the immunoassay of carcinoembryonic antigen: a feasibility study for clinical use, J. Nanobiotechnol., 2014, 12, 44.

30 C. C. Yang, S.-Y. Yang, H. H. Chen, W. L. Weng, H.-E. Horng, J.-J. Chieh, C. Y. Hong and H. C. Yang, J. Appl. Phys., 2012, 112, 012704.

31 M. K. Boehm and S. J. Perkins, Structural models for carcinoembryonic antigen and its complex with the singlechain Fv antibody molecule MFE23, FEBS Lett., 2000, 47511-47516.

32 K. Enpuku, T. Tanaka, T. Matsuda, F. Dang, N. Enomoto, J. Hojo, K. Yoshinaga, F. Ludwig, F. Ghaffari, E. Heim and M. Schilling, J. Appl. Phys., 2007, 102, 054901.

33 S. Y. Yang, J. F. Chang, T. C. Chen, C. C. Yang and C. S. Ho, Study of the temperature dependent immuno-reaction kinetics for the biofunctionalized magnetic nanoparticle assay of bio-markers of colorectal cancer, Appl. Phys. Lett., 2014, 104, 013702.

34 C. C. Yang, S. Y. Yang, J. J. Chieh, H. E. Horng, C. Y. Hong and H. C. Yang, IEEE Magn. Lett., 2012, 3, 1500104.

35 N. Gehlenborg, Comprehensive molecular characterization of human colon and rectal cancer, Nature, 2012, 487, 330337.

36 A. Bener, Colon cancer in rapidly developing countries: review of the life style, dietary, consanguinity and hereditary risk factors, Oncol. Rev., 2011, 5, 5-11. 